\title{
Effects of Carrier Frequency Accuracy on Quasi-Synchronous, Multicarrier DS-CDMA Communications Using Optimized Sequences
}

\author{
Kun-Wah Yip, Member, IEEE, and Tung-Sang Ng, Senior Member, IEEE
}

\begin{abstract}
It is known that the multiple access interference (MAI) of a quasi-synchronous (QS) multicarrier direct signal code division multiple access (DS-CDMA) system can be substantially reduced by using signature sequences having optimized cross correlation at small shifts around the origin. This paper shows that the time-frequency cross correlation function rather than the usual (time-domain) cross correlation determines the MAI when the system is operated in the presence of carrier frequency offset (CFO), which arises due to the frequencyaccuracy limit of the oscillator. Several known sets of sequences having optimized time-domain cross correlation are investigated for their MAI-minimization capabilities in the presence of CFO. It is found that: $i)$ a system using Walsh codes or Suehiro-Hatori polyphase sequences can be driven into outage as a result of significant worst-case MAI and ii) it is possible to minimize the MAI for systems using preferentially phased Gold codes cyclicshift $m$-sequences or Lin-Chang sequences only if the product of chip period and maximum frequency deviation is less than around 0.01. Implications of these findings to practical implementation of systems are discussed.
\end{abstract}

Index Terms - Carrier frequency offset (CFO), multicarrier direct signal code division multiple access (DS-CDMA), quasisynchronous (QS) transmission.

\section{INTRODUCTION AND MOTIVATION}

$\mathbf{I}$ $\mathrm{N}$ a wireless communication system, the carrier frequency of a transmitted signal is usually slightly off from the desired frequency because of the limit on the frequency accuracy of the oscillator. To date, typical oscillators have a frequency accuracy of around $\pm 10 \mathrm{ppm}$. As a result of the presence of carrier frequency offset (CFO), automatic frequency control (AFC) is usually implemented at the receiver. The AFC circuit adjusts the frequency of the receiver oscillator in order to synchronize it with the carrier frequency of the received signal.

In a direct sequence code division multiple access (DSCDMA) system, signals of different users are transmitted on the same frequency band, and each of these signals is subject to independent CFO. Since the receiver is intended to demodulate the desired signal only, the AFC circuit does not need to track the signals from other users so that the receiver oscillator is not frequency locked to the other user signals. As a result, the receiver is required to demodulate the desired signal in the

Manuscript received July 17, 1998; revised June 13, 1999. This work was supported in part by the Hong Kong Research Grants Council and by the University Research Committee at the University of Hong Kong, Hong Kong

The authors are with Department of Electrical and Electronic Engineering, University of Hong Kong, Hong Kong (e-mail: kwyip@hkueee.hku.hk; tsng@hkueee.hku.hk).

Publisher Item Identifier S 0733-8716(99)08270-0. presence of multiple access interference (MAI), whose carrier frequency is different from that of the desired signal. Although the presence of $\mathrm{CFO}$ in the MAI is a realistic phenomenon, its effects on the performance of DS-CDMA communications have only been investigated, to the best of authors' knowledge, in [1]-[4] for the case of asynchronous transmission. In this paper, we analyze a quasi-synchronous (QS) multicarrier DSCDMA system operating in the presence of CFO. We are particularly interested in the effects of CFO on the system performance when cross correlation-optimized signature sequences are used. To avoid confusion, we need to point out that there are three multicarrier-based CDMA techniques [5], namely, multicarrier CDMA, multicarrier DS-CDMA, and multitone CDMA. Despite similar names, they are very different in the transmission method. The scheme considered here is the second one. Description of multicarrier DS-CDMA systems can be found in [5], [6], and [8]. Before elucidating the significance of the present work and indicating the contribution of this paper, we briefly describe the motivation for considering QS multicarrier DS-CDMA communications.

DS-CDMA communications using quasi-synchronized signal transmission and cross correlation-optimized signature sequences have received considerable interest in past years because of the potential in supporting higher multiple access capacity, which is a desirable feature for future mobile and personal communication systems [7]-[9]. In a QS DS-CDMA system, the signals of all users are time aligned to within a small synchronization window, which is one or a few chip periods in length. The MAI is therefore determined by cross correlation at small shifts around the origin. By using signature sequences that have low cross correlation at small shifts around the origin, the MAI can be substantially reduced. Sets of sequences that possess such cross correlationoptimized property include, for example, the preferentially phased Gold code [7], the Walsh code [8], the set of cyclicshift $m$-sequences [9], polyphase sequence sets proposed by Suehiro and Hatori [10] and by Suehiro [11], and the set of sequences recently proposed by Lin and Chang [12]. Despite the advantage of reducing the MAI, one of the challenges in the implementation of a QS DS-CDMA system is to time synchronize signals of all users when the synchronization window is small. One approach for making implementation easier is to use multicarrier signaling to increase the chip period while maintaining the same data rate [8]. A longer chip period makes the synchronization window longer. 
In the presence of $\mathrm{CFO}$, it will be shown that the MAI of a QS multicarrier DS-CDMA system is determined by the time-frequency cross correlation [see (10)] rather than the usual (time-domain) cross correlation. The time-frequency cross correlation at zero time shift is reduced to the spectral cross correlation. The spectral cross correlation was introduced by Popović [13] in the study of multicarrier CDMA, a spread spectrum technique that spreads the signal in the frequency domain. Popović [13] also showed that for a set of sequences the behavior of spectral cross correlation is very different from that of the time-domain one. For example, orthogonality of Walsh codes in time-domain cross correlation is well known, but their normalized spectral cross correlation magnitude can be as high as unity [13], which indicates that the MAI of a synchronous multicarrier DS-CDMA system using Walsh codes and in the presence of CFO is very significant. In [7]-[12], the selection of optimized sequences was entirely based on minimization of the time-domain cross correlation. Since the CFO is inevitable in a QS multicarrier DS-CDMA system, it is important to know whether these optimized sequences can still minimize the MAI despite the presence of CFO. This knowledge is, however, not known in the previous literature. After developing a theoretical framework for analyzing QS multicarrier DS-CDMA communications in the presence of CFO, we investigate the MAI-minimization capabilities of these sets of optimized sequences.

Based on the system model described in Section II, we show that the MAI can be expressed in terms of the time-frequency cross correlation function in Section III. Time-frequency cross correlation properties of several known sets of sequences that are optimized in the time-domain cross correlation are studied in Section IV. In Section V, we derive: i) a bit error rate (BER) expression based on the Gaussian assumption of the MAI and ii) an upper and lower error bounds. Numerical results are given and used for studying effects of the CFO on the error performance. In Section VI, we discuss implications of our results and findings on practical systems. Finally, conclusions are drawn in Section VII.

\section{SySTEM MODEL}

The system under consideration consists of $K$ users. We consider multicarrier signaling that employs $M$ spectrally disjoint subcarriers with subcarrier spacing $\Delta f$. The bit stream of each user is first serial-to-parallel converted into $M$ substreams. Each substream is used to modulate a separate subcarrier using binary-phase shift keying, so that a total of $M$ bits are transmitted at a time. These $M$ bits are spread by the same signature sequence of length $N$. Let the bit transmission rate of each substream be $1 / T_{b}$ bits/s. The chip period $T_{c}$ is given by $T_{c}=T_{b} / N$.

The complex envelope of the $k$ th user transmitted signal is given by

$$
\begin{aligned}
s_{k}(t)= & \sqrt{2 P_{b}} e^{j\left(2 \pi \nu_{k} . t+\theta_{k}\right)} \\
& \times \sum_{n=-\infty}^{\infty} \sum_{m=0}^{M-1} b_{m, n}^{(k)} e^{j 2 \pi m \Delta f\left(t-\tau_{k}\right)} \phi_{k}\left(t-n T_{b}-\tau_{k}\right)
\end{aligned}
$$

where $P_{b}$ is the signal power per subcarrier, $\nu_{k}$ is the CFO of the $k$ th user signal, $\theta_{k}$ is the carrier phase which is modeled as a uniform random variable over $[0,2 \pi), b_{m, n}^{(k)}$, taking on a value of +1 or -1 with equal probabilities is the $n$th bit transmitted on the $m$ th subcarrier of the $k$ th user signal, $\tau_{k}$ is the time offset of the $k$ th user signal relative to a common time reference, and

$$
\phi_{k}(t)=\sum_{\ell=0}^{N-1} c_{\ell}^{(k)} \psi\left(t-\ell T_{c}\right)
$$

is the $k$ th user spectral spreading waveform. In (2), $\left\{c_{0}^{(k)}, \ldots, c_{N-1}^{(k)}\right\}$ is the $k$ th user signature sequence and $\psi(t)$ is the chip waveform satisfying $\int_{-\infty}^{\infty}|\psi(t)|^{2} d t=T_{c}$. We assume that $\left|c_{\ell}^{(k)}\right|=1$ and that the chip waveform is a square-root-raised-cosine pulse with a rolloff factor $\alpha$. Since the subcarriers are not spectrally overlapped, we set

$$
\Delta f=(1+\alpha) T_{c}^{-1} \text {. }
$$

Quasi-synchronized signal transmission is considered. We model that $\tau_{k}$ is uniformly distributed over $\left[-\Delta_{\tau} T_{c}, \Delta_{\tau} T_{c}\right]$ (so that $2 \Delta_{\tau} T_{c}$ is the size of the synchronization window) and that $\tau_{k}$ 's, $k=1, \ldots, K$, are mutually independent. In the special case that $\Delta_{\tau}=0$, signal transmission is perfectly synchronized. For the CFO, it is assumed that $\nu_{k}$ is uniformly distributed over $\left[-\Delta_{\nu}, \Delta_{\nu}\right]$, where $\Delta_{\nu}$ is the maximum frequency deviation from the desired carrier frequency and that $\nu_{k}$ 's are statistically independent. Although sophisticated physical models characterizing the frequency offset of an oscillator exist [14, ch. 2], the choice of this simple model is because our objective is to evaluate the effects of CFO on the performance, and this model enables us to accomplish this objective without excessive complexity. It is assumed that the carrier frequency is nearly constant over a duration of at least $T_{b}$ seconds so that demodulation of the signal is possible. This condition requires that the oscillator is temporally stable for at least $T_{b}$ seconds, and it is usually satisfied in practice.

We consider an additive white Gaussian noise (AWGN) channel with a two-sided power spectral density $N_{0} / 2$. The complex envelope of the received signal is given by

$$
r(t)=\eta(t)+\sum_{k=1}^{K} s_{k}(t)
$$

where $\eta(t)$ is the complex envelope of the AWGN. The receiver is intended to demodulate the $L$ th user signal. It is assumed that the receiver oscillator is able to frequencysynchronize at the $L$ th user signal, and a phase reference can be derived so that coherent detection is possible. The received signal is processed by a bank of matched filters, each of which detects the desired signal transmitted on a particular subcarrier. Without loss of generality we consider detection of $b_{\tilde{m}, 0}^{(L)}$. The matched filter output for decision making is given by

$$
\begin{gathered}
\hat{b}_{\tilde{m}, 0}^{(L)}=\frac{1}{\sqrt{2 E_{b} T_{b}}} \operatorname{Re}\left\{\int_{-\infty}^{\infty} r(t) e^{-j \theta_{L}-j 2 \pi\left[\nu_{L} t+\tilde{m} \Delta f\left(t-\tau_{L}\right)\right]}\right. \\
\left.\cdot \phi_{L}^{*}\left(t-\tau_{L}\right) d t\right\}
\end{gathered}
$$


where $(\cdot)^{*}$ denotes complex conjugate and $E_{b}=P_{b} T_{b}$ is the bit energy.

\section{ANALYSIS OF THE MAI}

We limit ourselves to consider only the case that $2 \Delta_{\nu}$, the maximum frequency difference between the carrier frequency of the desired signal and that of the other user signal, is less than $\Delta f$, the subcarrier frequency separation. Since subcarriers of a user's signal are assumed to be nonoverlapping, it follows that in the detection of the desired signal transmitted on the $\tilde{m}$ th subcarrier, the interference due to the other user signal is contributed only from its $\tilde{m}$ th subcarrier signal as well as from one of its adjacent subcarriers. While the condition $2 \Delta_{\nu}<\Delta f$ simplifies the analysis that follows, results obtained under this condition would be indicative to the situation when $2 \Delta_{\nu} \geq$ $\Delta f$. In the analysis, we do not consider the cases $\tilde{m}=0$ and $\tilde{m}=M-1$. In these two cases, it is possible that there is only one other user subcarrier signal that contributes to the MAI; in other cases, there are two. Therefore, these two cases may yield slightly optimistic results.

Define the time-frequency cross correlation function ${ }^{1}$ as

$C_{k, L}(\ell ; \mu)= \begin{cases}\sum_{i=0}^{N-1-\ell} c_{i}^{(k)} c_{i+\ell}^{(L)^{*}} e^{j 2 \pi i \mu}, & 0 \leq \ell \leq N-1 \\ \sum_{i=-\ell}^{N-1} c_{i}^{(k)} c_{i+\ell}^{(L)} e^{j 2 \pi i \mu,} & -(N-1) \leq \ell<0 \\ 0, & |\ell| \geq N .\end{cases}$

This function resembles the well-known ambiguity function in the radar literature [15, ch. 6.8]. It also reduces to the spectral cross correlation function [13] and the time-domain one in the special cases of $\ell=0$ and $\mu=0$, respectively. Note that $C_{k, L}(\ell ; \mu)$ is periodic in $\mu$ with a period of one. Let

$$
R(\tau ; \xi)=\frac{1}{T_{c}} \int_{-\infty}^{\infty} \psi(t) \psi^{*}(t+\tau) e^{j 2 \pi \xi t} d t .
$$

By taking Fourier transform on $R(\tau ; \xi)$ in the variable $\tau$, and since a square-root-raised-cosine pulse is considered, it can be shown that $R(\tau ; \xi)=0$ for $|\xi| \geq(1+\alpha) T_{c}^{-1}$. Let $n_{R}$ be a positive integer such that, for $|\xi|<(1+\alpha) T_{c}^{-1}, R(\tau ; \xi)$ is negligible when $|\tau|>n_{R} T_{c}$.

Substituting (4) into (5) yields

$$
\hat{b}_{\tilde{m}, 0}^{(L)}=\eta+b_{\tilde{m}, 0}^{(L)}+\sum_{k=1, k \neq L}^{K} J_{k}
$$

where $\eta$ is a zero-mean Gaussian random variable with a variance $\left(2 E_{b} / N_{0}\right)^{-1}$, and

$$
\begin{aligned}
J_{k}= & \frac{1}{T_{b}} \sum_{n=-\infty}^{\infty} \sum_{m=0}^{M-1} b_{m, n}^{(k)} \operatorname{Re}\left\{e^{j\left(\theta_{k}-\theta_{L}\right)}\right. \\
& \cdot \int_{-\infty}^{\infty} e^{j 2 \pi\left[\left(\nu_{k}-\nu_{L}\right) t+m \Delta f\left(t-\tau_{k}\right)-\tilde{m} \Delta f\left(t-\tau_{L}\right)\right]} \\
& \left.\cdot \phi_{k}\left(t-n T_{b}-\tau_{k}\right) \phi_{L}^{*}\left(t-\tau_{L}\right) d t\right\}
\end{aligned}
$$

\footnotetext{
${ }^{1}$ This is slightly different from the time-frequency cross correlation function that has also appeared in [13].
}

is the MAI due to the $k$ th user. After some algebraic manipulations, (9) can be expressed as

$$
\begin{aligned}
J_{k}=\operatorname{Re} & \left\{e^{j\left(\theta_{k}-\theta_{L}\right)+j 2 \pi\left[w_{k} \tau_{k}-\tilde{m}(\Delta f) \lambda_{k}\right]}\right. \\
& \cdot \sum_{i=-1}^{1} \sum_{n=-1}^{1} b_{i+\tilde{m}, n}^{(k)} e^{j 2 \pi\left(w_{k}+i \Delta f\right) n T_{b}} \\
& \cdot \sum_{q=-n_{q}}^{n_{q}} \frac{1}{N} C_{k, L}\left(n N+q ;\left(w_{k}+i \Delta f\right) T_{c}\right) \\
& \left.\cdot R\left(\lambda_{k}-q T_{c} ; w_{k}+i \Delta f\right)\right\}
\end{aligned}
$$

where

$$
\begin{aligned}
& w_{k}=\nu_{k}-\nu_{L} \\
& \lambda_{k}=\tau_{k}-\tau_{L}
\end{aligned}
$$

and

$$
n_{q}=\left\lceil n_{R}+2 \Delta_{\tau}\right\rceil-1 .
$$

Notice that $w_{k}$ and $\lambda_{k}$ follow triangular distributions over the ranges $\left[-2 \Delta_{\nu}, 2 \Delta_{\nu}\right]$ and $\left[-2 \Delta_{\tau} T_{c}, 2 \Delta_{\tau} T_{c}\right]$, respectively. From (10), it is clear that the MAI is characterized by the timefrequency cross correlation. In the special case that the signal transmission is perfectly synchronized $\left(\Delta_{\tau}=0\right)$, the MAI is solely determined by the spectral cross correlation. In the absence of $\mathrm{CFO}\left(\Delta_{\nu}=0\right)$, the time-domain cross correlation determines the resultant MAI.

It is insightful to consider the specific case that $\Delta_{\nu} \ll T_{c}^{-1}$. In this case, $\left|w_{k}\right|$ is much less than $T_{c}^{-1}$ so that $R\left(\lambda_{k}-\right.$ $\left.q T_{c} ; w_{k}+i \Delta f\right) \approx R\left(\lambda_{k}-q T_{c} ; i \Delta f\right), i=-1,0,1$. We also notice that most of the MAI is contributed from $b_{\tilde{m}, 0}^{(k)}$ because $\Delta_{\tau}$ is usually a small number as a result of quasi-synchronized signal transmission. Thus

$$
\begin{aligned}
J_{k} \approx \operatorname{Re} & \left\{e^{j\left(\theta_{k}-\theta_{L}\right)+j 2 \pi\left[w_{k} \tau_{k}-\tilde{m}(\Delta f) \lambda_{k}\right]} b_{\tilde{m}, 0}^{(k)}\right. \\
& \left.\cdot \sum_{q=-n_{q}}^{n_{q}} \frac{1}{N} C_{k, L}\left(q ; w_{k} T_{c}\right) \cdot R\left(\lambda_{k}-q T_{c} ; 0\right)\right\} .
\end{aligned}
$$

It is apparent that the MAI is determined by $C_{k, L}(\ell ; \mu)$ for $|\ell| \leq n_{q}$ and $|\mu| \leq 2 \Delta_{\nu} T_{c}$. Minimization of the MAI is therefore accomplished by using signature sequences (if they exist) that possess small $C_{k, L}(\ell ; \mu)$ magnitude over these ranges of $\ell$ and $\mu$. It is of particular interest to study the special case $\Delta_{\tau}=0.5$. This value of $\Delta_{\tau}$ has been recommended in a proposal of a satellite communication system using QS DS-CDMA [7]. If we assume that $R(\tau ; 0)$ is negligible for $|\tau|>T_{c}$ (usually justifiable for a moderate rolloff factor), then $J_{k}$ can be approximated by (14) with $n_{q}=1$. To minimize the MAI, we only need to select a set of sequences having small values of $\left|C_{k, L}(0 ; \mu)\right|$ and $\left|C_{k, L}(1 ; \mu)\right|, k \neq L$, over the range $|\mu| \leq 2 \Delta_{\nu} T_{c}$. We do not need to consider $\left|C_{k, L}(-1 ; \mu)\right|$ because $C_{k, L}(\ell ; \mu)=e^{-j 2 \pi \ell \mu} C_{L, k}^{*}(-\ell ;-\mu)$. 


\section{TIME-Frequency CROSS-CORRELATION Properties}

We now examine the time-frequency cross correlation properties of the following sets of optimized sequences that have been proposed in the literature. These sequences are optimized in the time-domain cross correlation.

1) Preferentially Phased Gold Codes [7]: These codes are a subset of Gold sequences having the property $C_{k, L}(0 ; 0)=-1, k \neq L$. We consider Gold codes of length $N=31$ generated from [7, Table I] characteristic polynomials $45_{8}$ (with initial phases $0_{8}-37_{8}$ ) and $67_{8}$ (initial phase $35_{8}$ ). A total of 32 sequences are obtained.

2) Walsh Codes: These codes are generated as rows of the Sylvester-Hadamard matrix. Here we consider Walsh codes of length $N=32$. A total of 32 different codes are generated.

3) Cyclic-Shift $m$-Sequences [9]: Consider an $m$-sequence $a_{0}, \ldots, a_{N-1}$. For a given shift $r$, construct the $k$ th user signature sequence by $c_{n}^{(k)}=a_{(n+k r) \bmod N}, n=$ $0, \ldots, N-1$. We can generate at most $\lfloor N / r\rfloor$ sequences using this method. These cyclic-shift $m$-sequences have the property that the periodic time-domain cross correlation is -1 for shifts within $r-1$. We study cyclic-shift m-sequences of length $N=31$ generated from the characteristic polynomial $51_{8}$ with $r=3$, so that a total of ten sequences are used.

4) Suehiro-Hatori Polyphase Sequences: A set of polyphase sequences having zero periodic time-domain cross correlation over all shifts has been proposed by Suehiro and Hatori [10]. A review on the construction of this sequence set is provided in [11]. Based on this sequence set, Suehiro [11] has proposed another sequence set having similar cross correlation properties. Owing to similarity of the two sequence sets, in this paper, we consider the set proposed in [10] only. The sequences we consider are columns of the matrix

$$
\frac{1}{\sqrt{3}} \mathbf{W}_{30}\left[\begin{array}{c}
\mathbf{I}_{10} \\
\mathbf{I}_{10} \\
W_{3} \mathbf{I}_{10}
\end{array}\right]
$$

where $\mathbf{W}_{30}$ is a $30 \times 30$ matrix given by $\mathbf{W}_{30}=$ $\left[W_{30}^{-m n}\right]_{m, n=0}^{29}, \mathbf{I}_{10}$ is a $10 \times 10$ identity matrix, and $W_{n}=\exp (j 2 \pi / n)$. The generated sequences have a length of $N=30$, and a total of ten sequences are formed.

5) Lin-Chang Sequences [12]: These sequences have periodic time-domain cross correlation value of -1 over a number of shifts. Since the construction method of this sequence set does not support generation of more than one sequence of length 31 , we consider sequences of length $N=63$. Five sequences are generated, based on the example given in $[12$, p. 225].

\section{A. Average Cross Correlation}

Let

$$
\Xi(\ell ; \mu)=\left[\frac{1}{N-|l|} \mathcal{E}_{\text {code }}\left(\left|C_{k, L}(\ell ; \mu)\right|^{2}\right)\right]^{1 / 2}, \quad|\ell| \neq N
$$

where $\mathcal{E}_{\text {code }}(\cdot)$ denotes averaging over all possible combinations of sequences, $k \neq L$, of the sequence set under consideration. In the special case that the sequences are binary valued and randomly generated, it can be shown that $\Xi(\ell ; \mu)=1$. Therefore, if the $\Xi(\ell ; \mu)$ value computed for the optimized sequence set under consideration is well below one over a given range of $\mu$, the optimizing property, and hence, the MAI-minimization capability of the sequence set are preserved in the presence of CFO. On the other hand, if $\Xi(\ell ; \mu)$ is close to unity, the sequences under consideration behave like random sequences in the presence of CFO and the capability to minimize the MAI due to optimized cross correlation is lost.

Fig. 1(a) plots the $\Xi(0 ; \mu)$ versus $\mu$ for $|\mu| \leq 0.1$. The deep notch around $\mu=0$ is a result of the cross correlationoptimized property that leads to substantial MAI reduction. However, the capability that the MAI can be substantially reduced diminishes rapidly when $|\mu|$ is away from the origin. The $\Xi(0 ; \mu)$ values of the Walsh code set, the sets of preferentially phased Gold codes, and cyclic-shift $m$-sequences approach one for $|\mu|$ as low as 0.02 , and for the Lin-Chang sequence set, $|\mu|=0.01$. For the Suehiro-Hatori sequence set, it has a relatively low $\Xi(0 ; \mu)$ for $\mu<0$, but $\Xi(0 ; \mu)$ is higher than one for positive $\mu$. In Fig. $1(\mathrm{~b})$, we plot $\Xi(1 ; \mu)$ versus $\mu$. It is apparent that $\Xi(1 ; \mu) \approx 1$ for the set of preferentially phased Gold codes, a result that arises because these codes are optimized only for $C_{k, L}(0,0)$ but not $C_{k, L}(1,0)$ [7]. It is also noticed that the $\Xi(1 ; \mu)$ values for all code sets, except the set of Suehiro-Hatori sequences, are close to unity for $|\mu| \geq 0.02$. This implies that the MAI-reduction ability is close to that of random sequences when $|\mu|$ is greater than 0.02 . Despite $\Xi(1 ; \mu)$ stays below 0.2 for the Suehiro-Hatori sequence set, the characteristic of its $\Xi(0 ; \mu)$ does not make this set useful for MAI minimization in the presence of CFO. Also, notice that Lin-Chang sequences are more sensitive to the CFO than Walsh codes, preferentially phased Gold codes and cyclic-shift $m$-sequences in the MAI-minimization capability as the Lin-Chang sequences have narrower notches in $\Xi(0 ; \mu)$ and $\Xi(1 ; \mu)$ around $\mu=0$.

To preserve the MAI-minimization capability of optimized sequences in the presence of $\mathrm{CFO}$, one needs to operate a system with $2 \Delta_{\nu} T_{c}$ (maximum possible value of $|\mu|$ ) less than around 0.02 (depending on the code set that is used). The significance of this $2 \Delta_{\nu} T_{C}$ value to practical implementation of QS multicarrier DS-CDMA systems is discussed in Section VI.

\section{B. Worst Case Scenario}

The worst-case MAI can be characterized by

$$
\Lambda(\ell ; \mu)=\frac{1}{N-|\ell|} \sup _{k \neq L}\left|C_{k, L}(\ell ; \mu)\right|, \quad \ell \neq N
$$

where the supremum is obtained by examining all possible combinations of sequences, $k \neq L$, of the sequence set that is considered. Fig. 2 plots the $\Lambda(0 ; \mu)$ for the sequence sets under consideration. The most important observation is that $\Lambda(0 ; \mu)$ attains a value of one at some values of $\mu$ for Walsh 


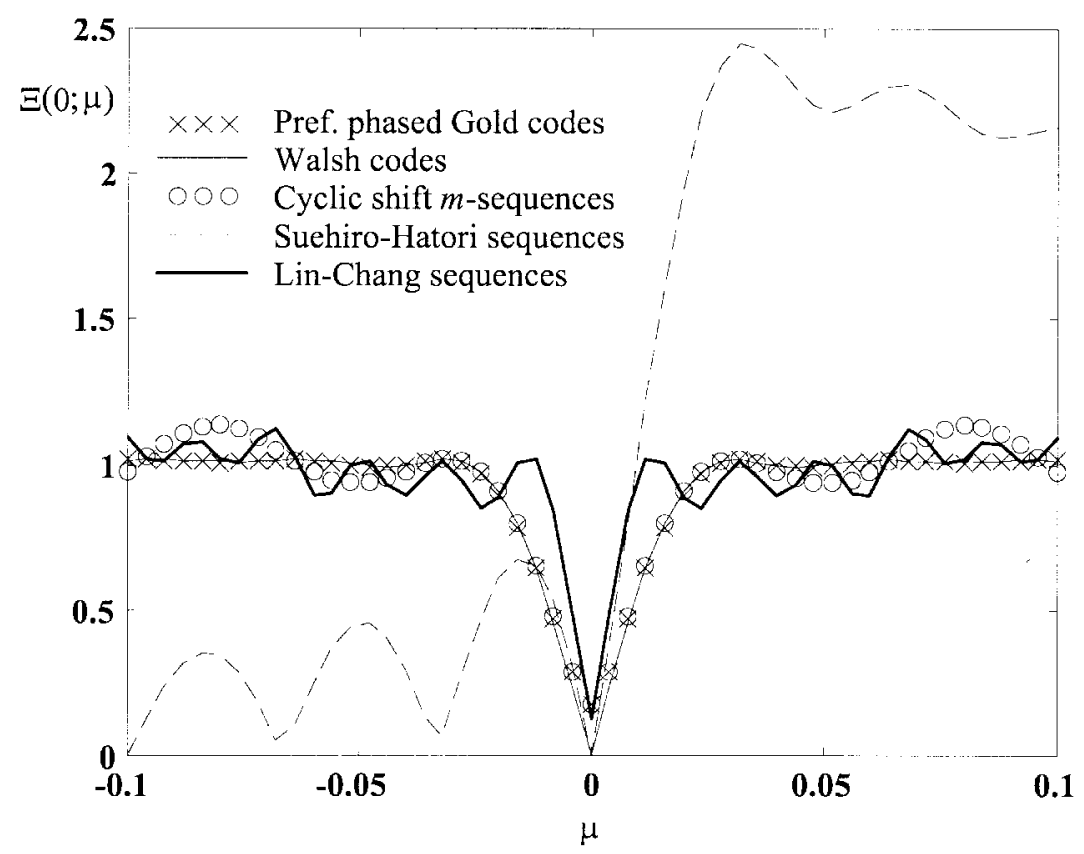

(a)

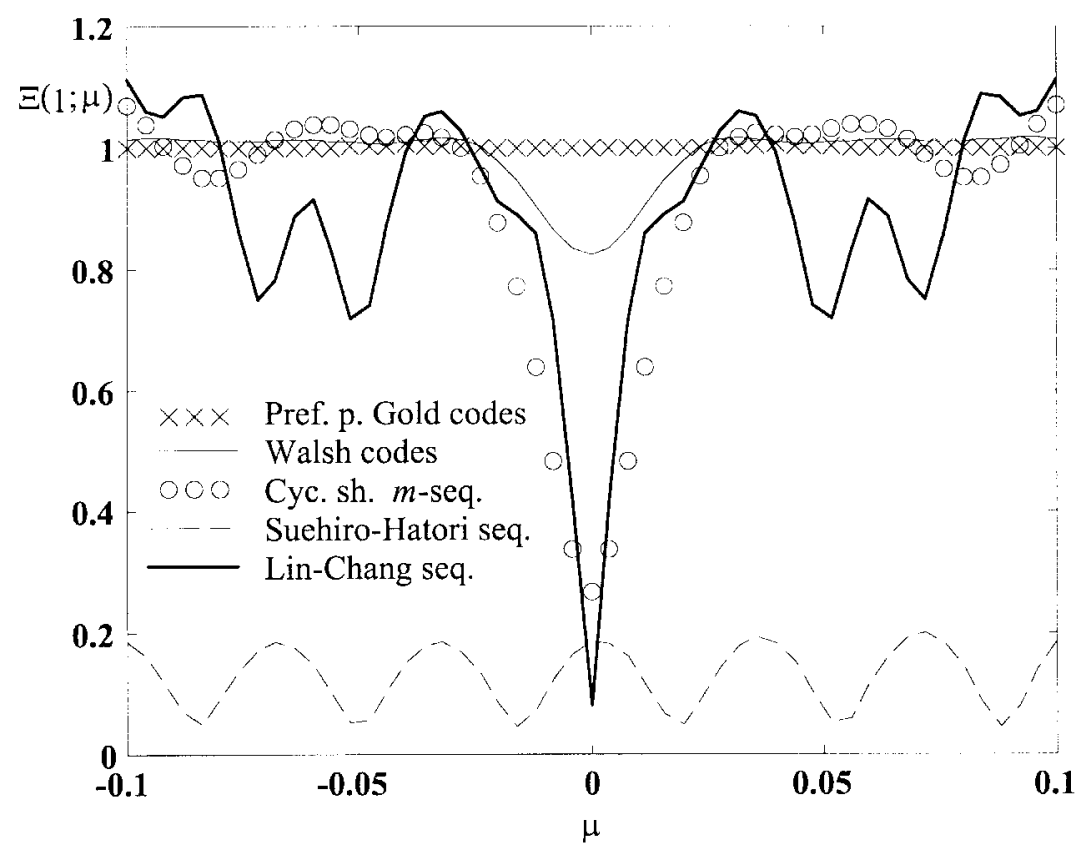

(b)

Fig. 1. Average time-frequency cross correlation: (a) $\Xi(0 ; \mu)$; (b) $\Xi(1 ; \mu)$. Note that the scales of $y$-axis for both subplots are not the same.

codes and Suehiro-Hatori polyphase sequences. (This result for the Walsh code set has also been reported by Popović [13] in the study of spectral cross correlation properties.) We also find that $\Lambda(1 ; \mu)$ shows a similar result of achieving a maximum value of one at some $\mu$ values, although we do not plot $\Lambda(1 ; \mu)$ here for saving space. Since $C_{k, L}(\ell ; \mu)$ is given by (6), our observation indicates that the $L$ th user sequence becomes identical to signature sequences of some of the other users after frequency and time shifting due to the CFO and quasi-synchronized signal transmission, respectively. Consequently, at some combinations of parameters the receiver may not be able to distinguish the desired signal against the
MAI when Walsh codes or Suehiro-Hatori sequences are used. In this situation, the system is driven into outage. As an example for illustration, consider a simple case that Walsh codes $\{+1,+1,+1,+1\}$ and $\{-1,+1,-1,+1\}$, respectively, are used as the $k$ th user and the $L$ th user signature sequences. Equation (6) indicates that, after frequency- and time-shifting, the $k$ th user and the $L$ th user sequences can be regarded as $\left\{c_{i}^{(k)} e^{j 2 \pi i \mu}\right\}_{i=0}^{N-1}$ and $\left\{c_{(i+\ell) \bmod N}^{(L)}\right\}_{i=0}^{N-1}$, respectively. In case $\ell=1$ and $\mu=0.5$, the two sequences after shifting become $\{+1,-1,+1,-1\}$, so that they are not distinguishable under these conditions for $\ell$ and $\mu$. Implications of the findings on the 


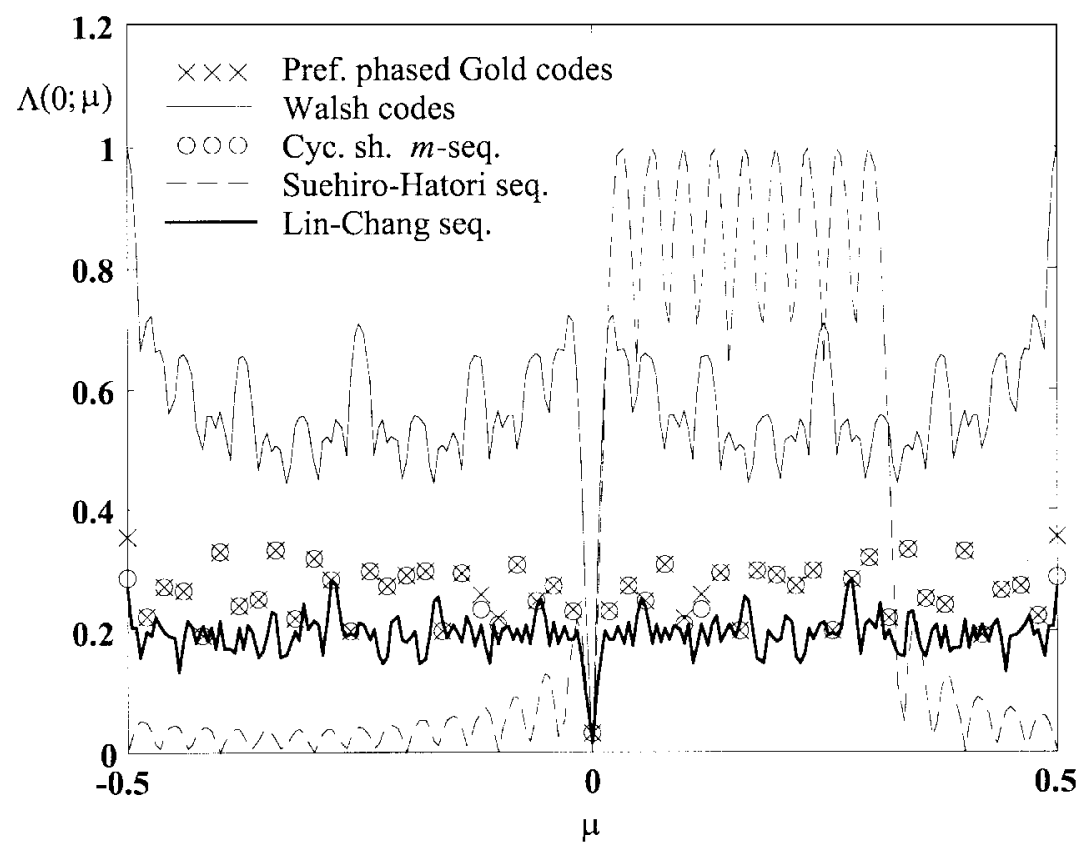

Fig. 2. Worst-case time-frequency cross correlation $\Lambda(0 ; \mu)$.

worst-case correlation to system implementation are explored in Section VI.

\section{IMPACTS OF THE CFO ON THE ERROR PERFORMANCE}

Although insightful results are obtained in Section IV based on the analysis of time-frequency cross correlation properties, it is desirable and useful to study the effects of CFO on the error performance. Before performing such analysis, we derive a BER expression based on the Gaussian assumption and check the accuracy of the resultant BER using error bounds.

\section{A. BER Expression}

We apply the Gaussian assumption on the distribution of the MAI. The accuracy of this assumption, which deserves particular attention, will be addressed later. Under this assumption, it follows from (8) that the BER $P_{e}$ is given by

$$
P_{e}=Q\left(\left\{\left(2 E_{b} / N_{0}\right)^{-1}+\sum_{k=1, k \neq L}^{K} \operatorname{var}\left(J_{k}\right)\right\}^{-1 / 2}\right)
$$

where $Q(\cdot)$ is the standard $Q$ function and $\operatorname{var}\left(J_{k}\right)$ is the variance of $J_{k}$. In this paper, we want to compute a representative BER for the whole set of sequences rather than for specific sequences. This is accomplished by averaging over all possible combinations of sequences $k \neq L$ in the computation of $\operatorname{var}\left(J_{k}\right)$. As a result, the $\operatorname{var}\left(J_{k}\right)$ values are equal for all $k$ 's, $k \neq L$. Let $\sigma^{2}=\operatorname{var}\left(J_{k}\right)$. It follows from (10) that

$$
\begin{aligned}
\sigma^{2}= & \frac{1}{2} \sum_{i=-1}^{1} \sum_{n=-1}^{1} \mathcal{E}_{\text {code }, \lambda_{\mathrm{k}}, \mathrm{w}_{\mathrm{k}}}\left\{\mid \sum_{q=-n_{q}}^{n_{q}} \frac{1}{N} C_{k, L}(n N+q ;\right. \\
& \left.\left.\left(w_{k}+i \Delta f\right) T_{c}\right)\left.\cdot R\left(\lambda_{k}-q T_{c} ; w_{k}+i \Delta f\right)\right|^{2}\right\}
\end{aligned}
$$

where $\mathcal{E}_{\text {code, } \lambda_{k}, w_{k}}\{\cdot\}$ denotes averaging over random variables $\lambda_{k}$ and $w_{k}$ and over all possible combinations of sequences, $k \neq L$. A double integral is involved in the averaging and numerical methods can be applied to compute $\sigma^{2}$. In the special case of random binary sequences, it can be shown that

$$
\sigma^{2}=\frac{1}{2 N} \sum_{i=-1}^{1} \sum_{q=-n_{q}}^{n_{q}} \mathcal{E}_{\lambda_{k}, w_{k}}\left\{\left|R\left(\lambda_{k}-q T_{c} ; w_{k}+i \Delta f\right)\right|^{2}\right\}
$$

where $\mathcal{E}_{\lambda_{k}, w_{k}}\{\cdot\}$ is the expectation over $\lambda_{k}$ and $w_{k}$. Substituting $\sigma^{2}=\operatorname{var}\left(J_{k}\right)$ into (17) gives

$$
P_{e}=Q\left(\left\{\left(2 E_{b} / N_{0}\right)^{-1}+(K-1) \sigma^{2}\right\}^{-1 / 2}\right) \text {. }
$$

BER computation using (20) has the advantage that $K$ needs not be an integer. Non-integral values of $K$ arise because we desire to fairly compare BER's of sequence sets under consideration; that is, we would like to compare $P_{e}$ under the same $K / N$ ratio.

\section{B. Error Bounds}

Accuracy of the Gaussian assumption is checked by comparing $P_{e}$ with the upper and lower error bounds that are derived as follows. Although these bounds are more accurate than the BER given by (20) because the Gaussian assumption is removed, they are not used in the rest of this paper because: i) huge computation is involved and ii) nonintegral values of $K$ cannot be handled.

The error bounds are derived based on the technique adapted from [16] and [17]. Since $J_{k}$ is expressed in terms of random variables $\lambda_{k}$, etc., one can obtain an approximate probability distribution function (PDF) of $J_{k}$

$$
\begin{aligned}
\operatorname{Pr}\left\{\left(i-\frac{1}{2}\right) \Delta_{J} \leq J_{k}<\left(i+\frac{1}{2}\right) \Delta_{J}\right\} & , \\
& i=-N_{J}^{(k)}, \ldots, N_{J}^{(k)}
\end{aligned}
$$


TABLE I

Comparison of BER $\left(P_{e}\right)$ AND CORRESPONDing UPPER $\left(P_{e}^{(U)}\right)$ AND Lower $\left(P_{e}^{(L)}\right)$ ERror Bounds for (a) Preferentially Phased Gold Codes, (b) Walsh Codes, (c) Cyclic-Shift $m$-Sequences, (d) Suehiro-Hatori Polyphase Sequences, and (e) Lin-Chang Sequences.

\begin{tabular}{ccccccc}
\hline Code set & $N$ & $K$ & $P_{e}$ & $P_{e}^{(L)}$ & $P_{e}^{(U)}$ & \\
\hline (a) & 31 & 6 & 1.93 & 1.49 & 1.61 & $\left(\times 10^{-4}\right)$ \\
(b) & 32 & 6 & 0.16 & 1.17 & 1.27 & $\left(\times 10^{-3}\right)$ \\
(c) & 31 & 6 & 1.42 & 0.97 & 1.05 & $\left(\times 10^{-4}\right)$ \\
(d) & 30 & 6 & 0.31 & 0.97 & 1.02 & $\left(\times 10^{-2}\right)$ \\
(c) & 63 & 13 & 5.08 & 5.70 & 6.03 & $\left(\times 10^{-4}\right)$ \\
\hline
\end{tabular}

by dividing random variables $\lambda_{k}$, etc., in small step size over their ranges and by means of counting. This approximate PDF is close to the actual one if $\Delta_{J}$ is sufficiently small. In obtaining the PDF of $J_{k}$, all possible combinations of signature sequences are examined. Let $J=\sum_{k=1, k \neq L}^{K} J_{k}$. The PDF of $J$

$$
\begin{aligned}
\operatorname{Pr}\left\{\left(i-\frac{1}{2}\right) \Delta_{J} \leq J<\left(i+\frac{1}{2}\right)\right. & \left.\Delta_{J}\right\}, \\
i & =-N_{J}, \ldots, N_{J}
\end{aligned}
$$

where $N_{J}=\sum_{k=1, k \neq L}^{K} N_{J}^{(k)}$, can be obtained by convolution. Since the BER conditioned on $(i-1 / 2) \Delta_{J} \leq J<(i+1 / 2) \Delta_{J}$ is upper and lower bounded by [see (8)] and consider $b_{\tilde{m}, 0}^{(L)}=$ 1)

$$
P_{e}^{(U)}(i)=Q\left(\sqrt{2 E_{b} / N_{0}}\left(1+\left(i-\frac{1}{2}\right) \Delta_{J}\right)\right)
$$

and

$$
P_{e}^{(L)}(i)=Q\left(\sqrt{2 E_{b} / N_{0}}\left(1+\left(i+\frac{1}{2}\right) \Delta_{J}\right)\right)
$$

respectively, it follows that the upper $\left(P_{e}^{(U)}\right)$ and lower $\left(P_{e}^{(L)}\right)$ error bounds are given by

$$
\begin{aligned}
\left\{\begin{array}{l}
P_{e}^{(U)} \\
P_{e}^{(L)}
\end{array}\right\}= & \sum_{i=-N_{J}}^{N_{J}} \operatorname{Pr}\left\{\left(i-\frac{1}{2}\right) \Delta_{J} \leq J<\left(i+\frac{1}{2}\right) \Delta_{J}\right\} \\
& \times\left\{\begin{array}{l}
P_{e}^{(U)}(i) \\
P_{e}^{(L)}(i)
\end{array}\right\} .
\end{aligned}
$$

\section{Numerical Results and Discussions}

Table I lists the values of $P_{e}$ computed by (20) and those of $P_{e}^{(U)}$ and $P_{e}^{(L)}$ obtained by using (23) for the sequence sets described in Section IV under the conditions $\Delta_{\tau}=0.5$, $\Delta_{\nu} T_{c}=0.1, E_{b} / N_{0}=15 \mathrm{~dB}$, and the rolloff factor $\alpha=0.5$. In the computation of numerical results, $n_{R}=5$ was used to compute $n_{q}$, given by (13). Note that the $K / N$ ratios are about $20 \%$ for all five sequence sets under consideration. Also notice that the Lin-Chang sequence set actually consists of five sequences only. The $P_{e}$ value for this sequence set was computed by substituting $K=13$ into (20) and, in the computation of $P_{e}^{(U)}$ and $P_{e}^{(L)}$, the PDF of $J$ was obtained by a 12-fold convolution on the PDF of $J_{k}$. It is apparent that the $P_{e}$ values are close to the error bounds for the preferentially phased Gold code set, the set of cyclic-shift $m$-sequences and the Lin-Chang sequence set, so that the Gaussian assumption yields close BER approximations for these three sets of codes.
However, overly optimistic results are obtained for the Walsh code set $\left(P_{e}\right.$ off from $P_{e}^{(U)}$ and $P_{e}^{(L)}$ for more than an order of magnitude) and Suehiro-Hatori sequence set (off for a factor of three). Therefore, the Gaussian assumption falls short for these two sets of codes.

Since (20) cannot give BER's with adequate accuracy for Walsh codes and Suehiro-Hatori polyphase sequences, we do not discuss the error performance for systems using these two sets of sequences. We are, however, more interested in the other three sets of codes because, as seen from the $P_{e}^{(U)}$ and $P_{e}^{(L)}$ values listed in Table I, they yield better error performance than the Walsh code set and the set of Suehiro-Hatori polyphase sequences under nearly the same $K / N$ ratio. From the system designers' point of view, the other three sets of codes are preferred. Therefore, not considering Walsh codes and Suehiro-Hatori sequences only results in a minor loss of contribution of this paper.

Fig. 3 plots the $P_{e}$ computed by (20) against $\Delta_{\nu} T_{c} \in[0,0.1]$ for preferentially phased Gold codes, cyclic-shift $m$-sequences and Lin-Chang sequences under the conditions $E_{b} / N_{0}=15$ $\mathrm{dB}, \alpha=0.5, n_{R}=5, \Delta_{\tau}=0.5$, and $K / N=20 \%$. The BER for random signature sequences is also plotted for comparison. We do not plot the BER for $\Delta_{\nu} T_{c}$ values greater than 0.1 as computation results, which are not shown here, indicate that over the range $\Delta_{\nu} T_{c} \in[0.1,0.5]$ the $P_{e}$ values lie within $1-5$ $\times 10^{-4}$ for the sequence sets under consideration, including the case of random sequences. We are more interested in the $P_{e}$ behavior at low $\Delta_{\nu} T_{c}$, as demonstrated by the results that follow.

Consider the three sets of optimized sequences. It is apparent that the BER's increase rapidly for $\Delta_{\nu} T_{C}$ increasing from zero. At large $\Delta_{\nu} T_{c}$, the BER's approach the $P_{e}$ value for random sequences. Since the BER's for the three sets of optimized sequences under consideration are of the order $10^{-4}$ at sufficiently large $\Delta_{\nu} T_{c}$ value, let us consider a BER of say $10^{-6}$ as the point below which the MAI-minimization capability of the sequence set under consideration is said to be preserved. It is found that $\Delta_{\nu} T_{c}=0.008,0.016$, and 0.007 for the set of preferentially phased Gold codes, the set of cyclic-shift $m$-sequences, and the Lin-Chang sequence set, respectively. These values of $\Delta_{\nu} T_{C}$ are consistent with the $\Delta_{\nu} T_{C}$ value $\left(2 \Delta_{\nu} T_{C} \approx 0.02\right)$ earlier found based on the analysis of the time-frequency cross correlation function in Section IV. It is also apparent that among the three sets of optimized sequences the MAI-minimization capability of the Lin-Chang sequence set is the most sensitive to the presence of CFO, a result that has also been found in Section IV.

It should be mentioned that our results have been obtained under the assumption $2 \Delta_{\nu}<\Delta f$ (Section III). We provide as follows a brief discussion on the error performance for a larger $2 \Delta_{\nu}$. When $2 \Delta_{\nu}<\Delta f$, the MAI is contributed only from the $(\tilde{m}-1)$ th, $\tilde{m}$ th and $(\tilde{m}+1)$ th subcarriers of the other user signal. For $2 \Delta_{\nu}>\Delta f$, the number of other user subcarrier signals involved for MAI contribution is more. However, at a particular frequency shift $w_{k}$, only two subcarrier signals are active for MAI contribution (provided $2 \Delta_{\nu}$ is less than $\tilde{m} \Delta f$ and $(M-1-\tilde{m}) \Delta f)$. It follows that the BER conditioned on $w_{k}$ is periodic with a period $\Delta f$. The desired BER is 


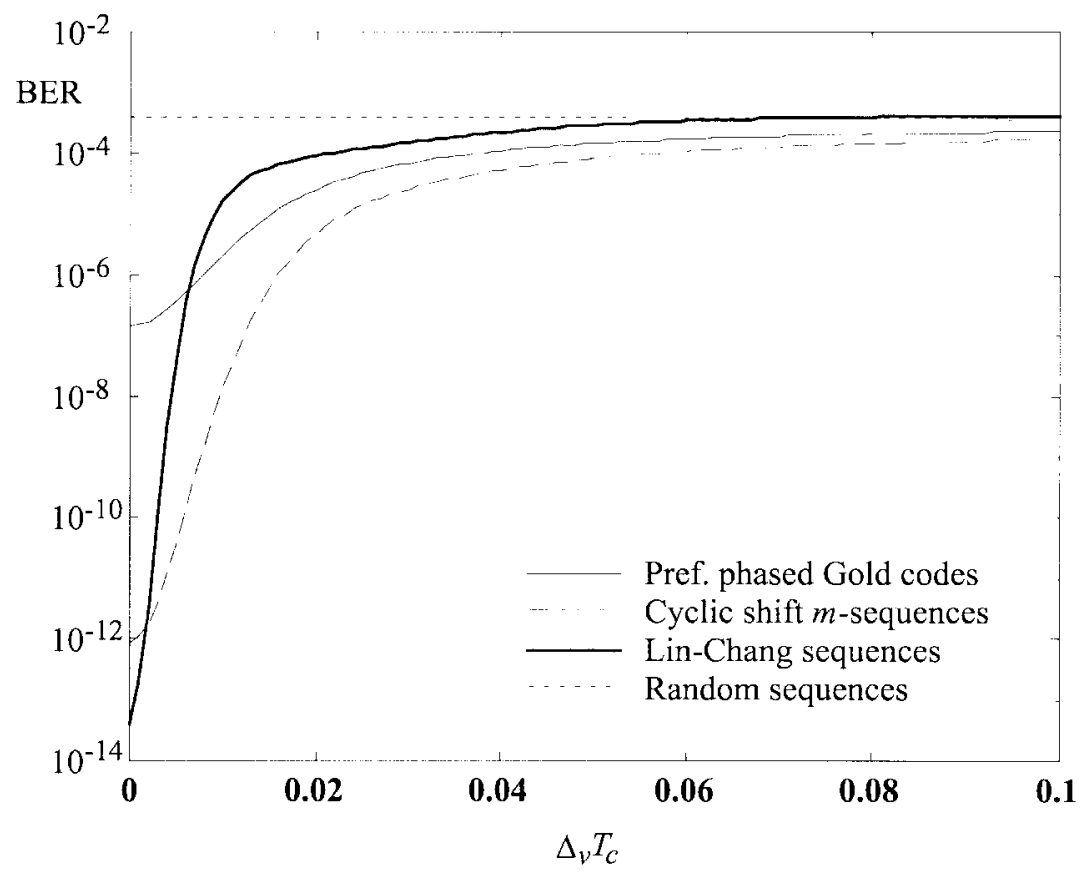

Fig. 3. BER versus $\Delta_{\nu} T_{C}$ at $E_{b} / N_{0}=15 \mathrm{~dB}, K / N=20 \%, \Delta_{\tau}=0.5$, and $\alpha=0.5$.

therefore obtained after averaging the conditional BER over the random variable $w_{k}=w_{k}(\bmod \Delta f)$. Since the BER for an optimized sequence set is close to that for random sequences unless the product of $w_{k} \bmod \Delta f$ and $T_{c}$ is very small, and since $w_{k}$ spans over $\left[-2 \Delta_{\nu}, 2 \Delta_{\nu}\right]$, it follows that resulted BER's for the three optimized sequence sets under consideration are close to the BER for random sequences when $2 \Delta_{\nu}>\Delta f$. Consequently, we conclude that the MAIminimization capabilities for these three sets of optimized sequences are limited only for small $\Delta_{\nu} T_{C}$ values.

\section{IMPLICATIONS TO SYSTEM IMPLEMENTATION}

Based on the analysis of the worst case time-frequency cross correlation in Section IV, it has been shown that the system using Walsh codes or Suehiro-Hatori polyphase sequences can be driven into outage at some combinations of parameters. The occurrence of outage is likely to result in a loss of communication link, which may lead to serious consequences for the system performance. Although Walsh codes and Suehiro-Hatori polyphase sequences show good timedomain cross correlation properties which have drawn much attention for applications to single-carrier and multicarrier QS DS-CDMA [8], [10], the systems implemented using these sequences are vulnerable to the presence of CFO.

Numerical results of Sections IV and V have indicated that it is possible to maintain the MAI-minimization capability of an optimized sequence set in the presence of CFO only if the system can be operated at a $\Delta_{\nu} T_{c}$ value less than around 0.01. Currently, the frequency accuracy of a typical frequency synthesizer is around $\pm 10 \mathrm{ppm}$. Let us consider a system operating at a desired carrier frequency of $1 \mathrm{GHz}$. It follows that $\Delta_{\nu}=10 \mathrm{kHz}$. One can utilize the MAIminimization capability of the optimized sequences only if the system can be operated at $T_{c}<1 \mu \mathrm{s}$. A higher carrier frequency results in a shorter $T_{c}$. However, a chip period of much longer than $1 \mu \mathrm{s}$ is generally desired for QS multicarrier DS-CDMA communications because of the consideration on the complexity involved in the time synchronization of all users' signals. Alternatively, one can operate a system with a tighter frequency control. For example, consider a system using $T_{c}=100 \mu \mathrm{s}$ and a carrier frequency of $1 \mathrm{GHz}$. Operating at $\Delta_{\nu} T_{C}<0.01$ requires that $\Delta_{\nu}<100 \mathrm{~Hz}$. Maintaining an oscillator running at a frequency of $1 \mathrm{GHz}$ with a frequency error less than $100 \mathrm{~Hz}$ is currently a challenging task.

\section{CONCLUSION}

We have shown that the MAI of a QS multicarrier DSCDMA communication system can be characterized by the time-frequency cross correlation function. Properties of this function have been analyzed for a number of sequence sets that are optimized in the time-domain cross correlation. It has been found that the Walsh code set and the set of Suehiro-Hatori polyphase sequences yield normalized worstcase cross correlation values equal to one. Thus, the system implemented using either of these two sequence sets can be driven into outage at some combinations of parameters. Analysis on the average time-frequency cross correlation has revealed that the optimized code sets we consider can maintain their MAI-minimization capabilities only if $\Delta_{\nu} T_{c}$ is less than 0.01. Numerical results have revealed that BER's for Walsh codes and Suehiro-Hatori sequences are higher than those for the preferentially phased Gold codes, the cyclicshift $m$-sequences and the Lin-Chang sequences under similar conditions. Based on the error performance analysis, it has been shown that MAI-minimization capabilities of the latter three sets of optimized sequences can be maintained only if $\Delta_{\nu} T_{C}$ can be kept at less than 0.007-0.016 (depending on the code set that is used). This range of $\Delta_{\nu} T_{C}$ is consistent with the 
one found in the analysis of the average time-frequency cross correlation. We have discussed the possibility of maintaining this $\Delta_{\nu} T_{C}$ requirement in practical system implementations. Discussion results have revealed that this requirement, which can be satisfied by operating a system with a shorter chip period or under a tighter frequency control, is currently still a challenging task.

\section{ACKNOWLEDGMENT}

The authors thank the anonymous reviewers for various useful comments and helpful suggestions.

\section{REFERENCES}

[1] E. S. Sousa, "The effect of clock and carrier frequency offsets on the performance of a direct-sequence spread-spectrum multiple-access system," IEEE J. Select. Areas Commun., vol. 8, pp. 580-587, May 1990.

[2] 2 , "Performance of a direct-sequence spread-spectrum multipleaccess system utilizing unequal carrier frequencies," IEICE Trans. Commun., vol. E76-B, pp. 906-912, Aug. 1993.

[3] P. V. Krishnamurthy and H. Leib, "DS-CDMA commutation signalling with frequency offsets for wireless communications," in Proc. 1995 Canadian Conf. Electrical Computer Engineering, Montreal, Quebec, Canada, pp. 404-407.

[4] T. Wada, T. Yamazato, M. Katayama, and A. Ogawa, "A new $M$ ary spread-spectrum multiple-access scheme in the presence of carrier frequency offset," in Proc. IEICE Trans. Fundamentals., vol. E79-A, pp. 1415-1422, Sept. 1996.

[5] S. Hara and R. Prasad, "Overview of multicarrier CDMA," IEEE Commun. Mag., vol. 35, pp. 126-133, Dec. 1997.

[6] S. Kondo and L. B. Milstein, "Performance of multicarrier DS CDMA systems," IEEE Trans. Commun., vol. 44, pp. 238-246, Feb. 1996.

[7] R. D. Gaudenzi, C. Elia, and R. Viola, "Bandlimited quasi-synchronous CDMA: A novel satellite access technique for mobile and personal communication systems," IEEE J. Select. Areas Commun., vol. 10, pp. 328-343, Feb. 1992.

[8] V. M. DaSilva and E. S. Sousa, "Multicarrier orthogonal CDMA signals for quasi-synchronous communication systems," IEEE J. Select. Areas Commun., vol. 12, pp. 842-852, June 1994.

[9] K. W. Yip and T. S. Ng, "Code phase assignment-A technique for highcapacity indoor mobile DS-CDMA communications," in Proc. VTC'94, Stockholm, Sweden, pp. 1586-1590.

[10] N. Suehiro and M. Hatori, "Polyphase periodic sequences without cross correlation and their application to asynchronous SSMA systems," in Proc. IEICE Trans., vol. J68-A, Oct. 1985, in Japanese.

[11] N. Suehiro, "A signal design without co-channel interference for approximately synchronized CDMA systems," IEEE J. Select. Areas Commun., vol. 12, pp. 837-841, June 1994.

[12] X. D. Lin and K. H. Chang, "Optimal PN sequence design for quasisynchronous CDMA communication systems," IEEE Trans. Commun., vol. 45, pp. 221-226, Feb. 1997.

[13] B. M. Popović, "Spreading sequences for multi-carrier CDMA systems," in Proc. Inst. Elect. Eng. Colloquium CDMA Techniques and Applications for Third Generation Mobile Systems, London, U.K., May 1997, pp. $8 / 1-8 / 6$.
[14] U. L. Rohde, Microwave and Wireless Synthesizers. New York: Wiley, 1997.

[15] P. Z. Peebles Jr., Radar Principles. New York: Wiley, 1998.

[16] J. S. Lehnert, "An efficient technique for evaluating direct-sequence spread-spectrum multiple-access communications," IEEE Trans. Commun., vol. 37, pp. 851-858, Aug. 1989.

[17] K. W. Yip and T. S. Ng, "Tight error bounds for asynchronous multicarrier CDMA and their application," IEEE Commun. Lett., vol. 2, pp. 295-297, Nov. 1998.

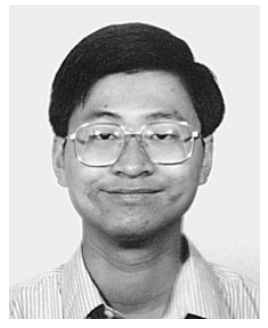

Kun-Wah Yip (M'96) received the B. Eng. degree (with honors) from the University of Bradford, U.K., in 1991 and the Ph.D. degree from the University of Hong Kong, Hong Kong, in 1995, both in electrical engineering.

From 1995 to 1998, he was a Research Associate and then a Postdoctoral Fellow at the University of Hong Kong. Currently, he is a Research Assistant Professor at the same university. His research interests are in spread-spectrum communications, multicarrier signal transmission, efficient simulation techniques, and theory and techniques for communications over frequencyselective fading channels.

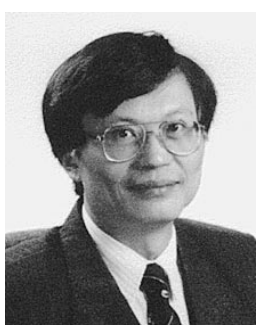

Tung-Sang Ng (S'74-M'78-SM'90) received the B.Sc. degree from the University of Hong Kong, Hong Kong, in 1972, and the M.Eng.Sc. and Ph.D. degrees from the University of Newcastle, Australia, in 1974 and 1977, respectively, all in electrical engineering.

He worked for BHP Steel International and the University of Wollongong, Australia, after graduation for 14 years and returned to the University of Hong Kong in 1991, taking up the position of Professor and Chair of Electronic Engineering. His current research interests include mobile communication systems, spreadspectrum techniques, CDMA, and digital signal processing. He has published over 170 international journal and conference papers. He is currently a Regional Editor of the International Journal Engineering Applications of Artificial Intelligence.

Dr. Ng was awarded the Honorary Doctor of Engineering Degree by the University of Newcastle, Australia, in 1997, for his services to higher education generally and to engineering education specifically. He was the General Chair of ISCA'97 and is currently VP-Region 10 of IEEE Circuits and Systems Society. He is also an Executive Committee Member and a Board Member of the Institute of Electrical Engineering's Informatics Divisional Board. He is a Fellow of the Institute of Electrical Engineering, HKIE, and IEAust. 